\title{
Unlocking of the Orthopedic Department in the Largest Tertiary Referral Hospital of Iran: Our Experience during Coronavirus Disease 2019 Pandemic
}

\author{
Seyed Hadi Kalantar $₫$, Seyed Mohammad Javad Mortazavi, Mohammadreza Razzaghof $\mathbb{D}$, \\ Alireza Moharrami, Mohammad Zarei, Nima Bagheri
}

Joint Reconstruction Research Center, Tehran University of Medical Sciences, Tehran, Iran

Corresponding author: Mohammadreza Razzaghof; Joint Reconstruction Research Center, Tehran University of Medical Sciences, Tehran, Iran. Tel: +98-9134610054, Email:m.razzaghof@gmail.com

Received: 17 February 2020; Revised: 12 April 2020; Accepted: 19 May 2020

\begin{abstract}
Background: The novel severe acute respiratory syndrome coronavirus 2 (SARS-CoV-2) has caused the largest global healthcare crisis of the last century since its emergence in December 2019. As more of the condition's chronicity became evident and the government policies changed, the unlocking of the orthopedic wards seemed necessary. In this study, we decided to share our experience regarding the unlocking of our orthopedic ward in the largest tertiary referral hospital of Iran and to explain its negative and positive consequences.

Methods: This observational study was done in Imam Khomeini Hospital Complex, Tehran, Iran, from April to August 2020. We extracted the data regarding the number of elective/trauma operations, emergency patients, and morning report sessions attendance from our registry database. The data were analyzed and plotted using Microsoft Excel 365.

Results: The mean weekly number of orthopedic operations decreased from 50 for elective and 30 for trauma operations in January 2020 to almost zero for both in late March and early April 2020 [the first coronavirus disease 2019 (COVID-19) peak]. Following the department unlocking, the mean weekly elective and trauma operations returned to 50 and 20 by mid-June, respectively. The mean weekly orthopedic emergency visits followed the same order with decrease from 50 to almost zero in late March and increase to 70 in mid-June. However, by the beginning of the second wave, it decreased to almost 38 in early July and further to 28 in late July by the end of the second wave.

Conclusion: The unlocking of the orthopedic department in a tertiary referral hospital during the COVID-19 pandemic is possible, provided that there is a set of protocols for patient care in the ward, emergency room(ER), operating room(OR), and clinic during the pandemic.
\end{abstract}

Keywords:Coronavirus; COVID-19; Pandemic; Orthopedics

Citation: Kalantar SH, Mortazavi SMJ, Razzaghof M, Moharrami A, Zarei M, Bagheri N. Unlocking of the Orthopedic Department in the Largest Tertiary Referral Hospital of Iran: Our Experience during Coronavirus Disease 2019 Pandemic. JOrthop Spine Trauma 2020; 6(2):33-8.

\section{Background}

The novel severe acute respiratory syndrome coronavirus 2 (SARS-CoV-2) has caused the largest global healthcare crisis of the last century since its emergence in Wuhan, China, in December 2019 (1-3). It was announced as a pandemic by the World Health Organization (WHO) on March 11, 2020 (4). To date (August 28, 2020), 24587513 confirmed cases and 833556 deaths have been reported worldwide (5). Iran was one of the first countries affected by the disease (6). A number of 369911 confirmed cases and 21249 deaths has been reported so far, which puts Iran in the first rank of total confirmed cases in the WHO Eastern Mediterranean Region (EMRO) and in the $12^{\text {th }}$ rank worldwide (5). At the very moment, more than one Iranian is getting the disease every minute and, one Iranian is dying every 10 minutes due to coronavirus disease 2019 (COVID-19) infection (5).

Since the very emergence of the pandemic, it imposed a massive burden on the health sector and all aspects of medical practice, including even those specialties not directly related to the outcomes of COVID-19 infection like orthopedic surgery (7). Iran was not an exception, and our orthopedic surgery community attempted to help in handling the crisis by developing protocols and instructions to modify the management of orthopedic patients and accommodate it to the new circumstances (8-10). This resulted in a substantial decrease in the rate of elective orthopedic surgeries and the allocation of orthopedic wards and healthcare staff to COVID-19 patients. The orthopedic operating rooms (ORs) were only open to emergent and urgent trauma patients $(11,12)$. Moreover, in university hospitals, the training of orthopedic surgery residents and medical students was almost ceased (11).

As more of the condition's chronicity became evident and the government policies changed from strict quarantine and community containment rules to self-isolation and social distancing, the reopening and unlocking of the orthopedic wards and activities seemed necessary (13-15). It was particularly because many orthopedic patients with semiurgent and chronic conditions required surgery as they had been postponed since the beginning of the pandemic (15). Furthermore, with the downslope of the COVID-19 infection rate, the educational activities needed to resume, as they have been almost limited to tele-education and webinars with no place for practice-based learning for nearly four months since February 2020. In this review study, we decided to share our experience regarding the unlocking of our orthopedic ward in one of the largest tertiary referral hospitals of Iran and to explain its negative and positive consequences. 


\section{The Characteristics of Our Department}

Our orthopedic department is placed in Imam Khomeini Hospital Complex (Tehran, Iran), which is the largest general tertiary referral hospital of the country with more than 1300 active beds and almost all the medical subspecialties (16). Our department is an orthopedic referral center in the country and has nearly all the orthopedic subspecialties, including arthroplasty, tumor, spine, sports, shoulder, foot and ankle, and hand surgery. There is also a trauma section for orthopedic trauma patients. We have a specialized orthopedic OR complex with six active ORs, in which an average of 460 orthopedic operations, 360 elective and 100 trauma cases, were performed monthly before the beginning of the COVID-19 pandemic.

\section{A Brief Report on the Lockdown Period}

With our hospital becoming one of the leading referral centers for admitting COVID-19 patients, the hospital policy was not to admit trauma patients. Our department also decided to postpone all the elective surgeries. Therefore, our monthly operation rate decreased from 360 elective and 100 trauma surgeries to almost zero in the late February and March 2020. The only orthopedic surgeries performed during this time was limited to necessary amputations for diabetic feet and arthrotomies for septic arthritis, which also decreased by $90 \%$. The orthopedic interns and students were discharged, and the resident shifts were reduced so that they could take part in the corona treatment team. We also used telecommunication and virtual office system to communicate with the patients for their follow-up. The educational programs carried on in the form of daily virtual morning reports and weekly journal club/case presentation webinars (11).

\section{The Unlocking of Our Department}

As the downslope of COVID-19 incidence began in Iran by early April and as the disease showed no imminent sign of leaving the community, the strict prohibitions on social activities and interactions were modified, and the small and large businesses came back to work. Nevertheless, the social distancing protocols were continued. Those medical subspecialty fields like orthopedics, which were unrelated to COVID-19 patients' care and had almost completely stopped their activities to make their facilities and staff available to COVID-19 patients, were not an exception in this process. On the one hand, our hospital policy changed to readmit the trauma patients from the Emergency Medical Service (EMS) and our department turned back into its previous state of a "trauma center". On the other hand, there was an increasing demand from patients with chronic disabilities and non-urgent conditions, whose elective surgeries had been postponed due to the COVID-19 epidemic for nearly four months. These included mostly the patients requiring hip or knee arthroplasty, musculoskeletal tumor surgery, and pediatric surgery with a time limit. As a result, our wards and ORs were to be reopened, and our clinics were to accept new patients; as formerly, only follow-up patients had been visited in our clinics. However, in advance, we required a set of protocols for the OR, orthopedic wards, orthopedic clinics, and emergency room (ER). After multiple consulting sessions, these protocols were established by the head of our department, the orthopedic and anesthesiology faculties at our institute, and an infectious disease specialist.

\section{Methods}

This observational study was done in Imam Khomeini
Hospital Complex from April to August 2020. Our primary outcomes were the number of elective and trauma orthopedic operations, the number of emergency orthopedic patients, and the number of faculties, residents, and fellowships attending the morning sessions. We extracted these data from our registry of operations, emergency visits, and morning report attendance list. The data were analyzed using Microsoft Excel 365. Bar and line charts were used to depict data along the time. We reported the number of operations, emergency patients, and morning report attendance on a daily, weekly, and monthly basis. The protocols of unlocking used in our study were established after multiple consulting sessions with the presence of the head of our department, the orthopedic and anesthesiology faculties at our institute, and an infectious disease specialist. The Unlocking Protocols

In general, the orthopedic operations performed in our institute are one of the two types: elective and trauma. Since the beginning of the pandemic, our hospital allocated a special unit with the help of emergency medicine and infectious diseases services to screen all the patients who enter the hospital for either elective or emergency care, for COVID-19. The screening is done by taking history of COVID-19-related symptoms and signs, checking vital signs and pulse oximetry, and physical examination. Any elective patient who has symptoms and signs suspicious for COVID-19, oxygen saturation of less than $93 \%$, or fever (temperature $>38^{\circ} \mathrm{C}$ ) should undergo further radiologic and laboratory work-up for COVID-19 and cannot be admitted unless COVID-19 is ruled out. Those patients in need of emergent care are admitted in the emergency ward allocated to COVID-19 and undergo COVID-19 work-up while receiving their required emergent care. We performed no routine chest x-ray, high-resolution computed tomography (HRCT) chest scan, COVID-19 polymerase chain reaction (PCR) test, and antibody screening. As a result, all our patients undergo the necessary basic screening and are separated prior to admission. Nevertheless, a set of protocols were required for the clinics, ER, wards, and ORs. The specific protocols related to each section are presented below.

ER Protocols

1. The orthopedic residents and interns are not required to be physically present all the time; rather they should be prepared on call when a patient needs orthopedic attention/treatment.

2. The orthopedic interns and residents should use the standard personal protective equipment (PPE) during their ER attendance. In this regard, all the patients should be considered possible silent carriers, and the basic PPE, including wearing a surgical mask, medical gown, and gloves during the visits, physical examination, and performing procedures are mandatory.

3. The orthopedic room should be disinfected frequently, at least every four hours daily, according to the WHO guideline on "cleaning and disinfection of environmental surfaces in the context of COVID-19" (17).

4. The patients needing surgery should undergo necessary preoperative work-up and transferred to OR as soon as possible to minimize the ER admission time.

5. The patients needing longer admission, who are suspicious for COVID-19, should be transferred to the wards allocated to COVID-19 care, and not the orthopedic ward.

6. Those trauma patients with relative indications of surgical treatment, that could be managed non- 
operatively, should undergo non-operative treatment to reduce the workload on the OR and staff, and avoid patients' crowding at the OR.

Orthopedic Clinics Protocols

1. As our clinic is usually busy with a range of 40-120 patients on different days, we set a maximum limit of 30 patients daily to avoid the patients' crowding in the clinic.

2. Our clinic is composed of a room divided by paravans into six compartments for visiting the patients. During COVID-19, we decided that only half of the compartments be active simultaneously.

3. The patients are allowed to enter the room alone with no companion, if possible. For children or the disable patients, who need someone's help, only one company is allowed.

4. No patient is allowed to enter the room before the previous patient leaves it.

5. All the physicians, staff, and patients should use the standard PPEs, including at minimum wearing a surgical mask and disposable gloves.

6. The physician should disinfect their hands or change their gloves after examining each patient.

7. Those follow-up visits which do not require the physical presence of the patients are done through the virtual clinic.

\section{Orthopedic Wards Protocols}

1. As mentioned above, all the elective and trauma patients could be admitted to the orthopedic ward only if they had gone through the screening schedule performed by the special unit on hospital entry. Our head-nurse checked the patient's COVID-19 screening sheet and it should have been 'negative' to sign the patient's admission card.

2. The distance between adjacent patient beds should be 2 meters.

3. All the healthcare staff of the ward must use the standard PPE.

4. If a patient becomes suspicious or diagnosed with COVID-19 after his admission to the orthopedic ward, he must be transferred to COVID-19 wards for further work-up and treatment.

5. The trauma and elective patients should be discharged as soon as possible, e.g., on the same or next postoperative day. Practically, only the major trauma, e.g., pelvic, spine, long bone fractures, or multiple trauma patients with multiple fractures or associated brain/spinal cord or internal organs trauma, were excluded.

6. The wards must be disinfected frequently, at least every four hours, according to the WHO guideline on "cleaning and disinfection of environmental surfaces in the context of COVID-19" (17).

\section{ORs Protocols}

\section{COVID-19 Trauma Patients}

1. Every day, one or two OR rooms are allocated to only COVID-19 trauma patients. No COVID-19-negative patient was transferred to these rooms on that day.

2. At first, the anesthesiology team enter the room with the minimum possible members and anesthetize the patient. They must be provided with standard PPE for COVID-19-positive patients, including at least an N-95 mask, face shield, medical gown, and gloves. During inducing the anesthesia, the surgery team is not allowed in the room.

3. After the anesthesia is done, the surgery team will enter the room with the minimum possible members. They must be provided with standard PPE for COVID-19positive patients, including at least an N-95 mask, face shield, medical gown, and gloves.

4. The OR must be disinfected completely after the surgery is completed, and the patient leaves the room.

5. The COVID-19 patients are not transferred to the recovery room. The recovery process is done in the OR, and the patient is transferred directly to the COVID-19 ward.

6. The used surgical instruments are labeled as " COVID-19", so that the disinfecting personnel become well aware of that and use the standard needed PPE.

Non-COVID-19 Patients

1. The surgeries should be performed with the minimum possible persons. As a result, no extra person, e.g., the students and interns, is allowed in the OR.

2. The OR must be disinfected completely between operations and before complete disinfection, the next patient is not allowed in that room.

3. The surgeons, anesthesiologists, and nurses are provided with standard PPE, including surgical masks, face shields, medical gowns, and gloves.

Recovery Room

1. The distance between patient beds must be at least 2 meters.

2. The anesthesiologist should attempt to transfer the patients to their wards as soon as possible to avoid crowding in the recovery room.

3. All the healthcare staff should be provided with the standard PPE.

4. The recovery room should be equipped with a negative ventilation system, if possible. Otherwise, the appropriate ventilation should be present in the recovery room.

\section{Educational Protocols}

As education is an inseparable part of our system in a high-grade university hospital and postponing the educational programs for long will seriously damage the training of the orthopedic residents, we resumed our educational programs, including the morning reports and educational classes, hence the theoretical training, besides reactivating the ORs, hence the practical training. Despite continuing the weekly journal clubs and case presentations through virtual webinars, our daily morning reports and educational classes were held in-person. It was because all the residents should have attended their shifts in the orthopedic wards, OR, ER, and clinics, and it was easier and more efficient for them to attend the classroom in person. The faculties should have also attended the classes. Our protocols for running the classroom were as follows:

1. There must be a longitudinal and horizontal distance of at least 2 meters between adjacent persons.

2. Those residents or faculties, who were suspicious or diagnosed with COVID-19, were prohibited from attending the hospital for at least two weeks after being symptom-free.

3. The air conditioners and ventilations must be active during the class time.

4. The classroom, including all the chairs, desks, laptop, and computer systems, must be disinfected every day after the class is finished according to the WHO guideline on "cleaning and disinfection of environmental surfaces in the context of COVID-19" (17).

5. All the attending residents and faculties should use the standard PPE, including at least a surgical mask, during the class time.

\section{Results}

Applying the aforementioned protocols, our orthopedic 
department was unlocked and resumed its activity since April 2020. The mean number of orthopedic operations, both elective and trauma, and the mean number of emergency orthopedic patients' visits, both trauma and soft tissue infections, incremented to its previous level. Figure 1 shows the mean weekly number of elective and trauma orthopedic operations superimposed on the chart of the daily new cases of COVID-19 in Iran. As can be seen, by the beginning of the COVID-19 epidemic in early February 2020, the mean weekly number of orthopedic operations decreased from 50 for elective and 30 for trauma operations in January 2020 to almost zero for both in late March and early April 2020, simultaneous with the first peak of COVID19 in Iran (3070 new daily cases). However, in the downslope of the first wave, i.e., late April, we unlocked our department, which was concurrent with unlocking many other small and large businesses in the country. From that time, as the charts show, the mean weekly number of elective and trauma operations returned to its previous rates of nearly 50 and 20 by mid-June, respectively. In this time, two inauspicious events happened. First, the second wave and peak of COVID-19 occurred throughout the country, which was much more vigorous than the first one. It resulted in a decrease in both the number of elective and trauma patients. Secondly, a considerable proportion of our residents, faculties, and OR staff got infected with COVID-19. As a result, the mean weekly number of both elective and trauma operations decreased to almost ten simultaneous with the second peak of COVID-19 in Iran by mid-July ( 3500 new daily cases). This situation carried on for almost three weeks and resolved with the recovery of almost all of the infected residents and OR staff. Thus, we returned to and even surpassed our previous weekly rate of operations, that is, 60 elective and 30 trauma operations.

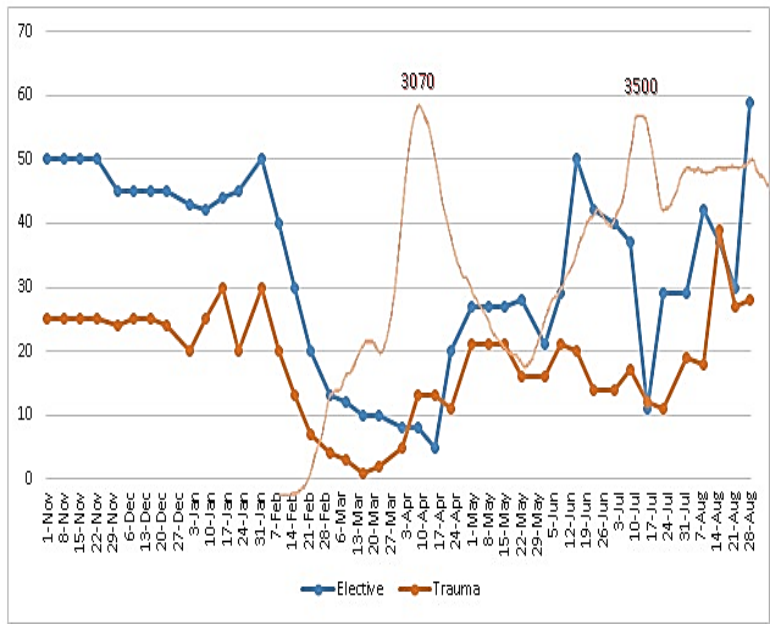

Figure 1. The mean weekly number of elective and trauma orthopedic operations superimposed on the chart of the daily new cases of coronavirus disease 2019 (COVID-19) in Iran

Figure 2 depicts the mean weekly number of orthopedic patients visited in the ER against the chart of the daily new cases of COVID-19 in Iran. This chart also follows the pattern of the previously-discussed chart of the mean weekly number of operations. As it is evident, the mean weekly orthopedic emergency visits decreased from 50 to almost zero in late March, simultaneous with the first COVID-19 peak. Concurrent with the first wave's downslope in late April, the weekly number of orthopedic emergency visits incremented to 70 in mid-June. However, by the beginning of the second wave, it decreased to almost 38 in early July and further to 28 in late July by the end of the second wave. It was also concurrent with the period during which most of our residents got infected with COVID-19. Thereafter, the mean weekly number of emergency visits increased to its previous number of 50 in early August and even more, i.e., 70-80 in mid to late August.

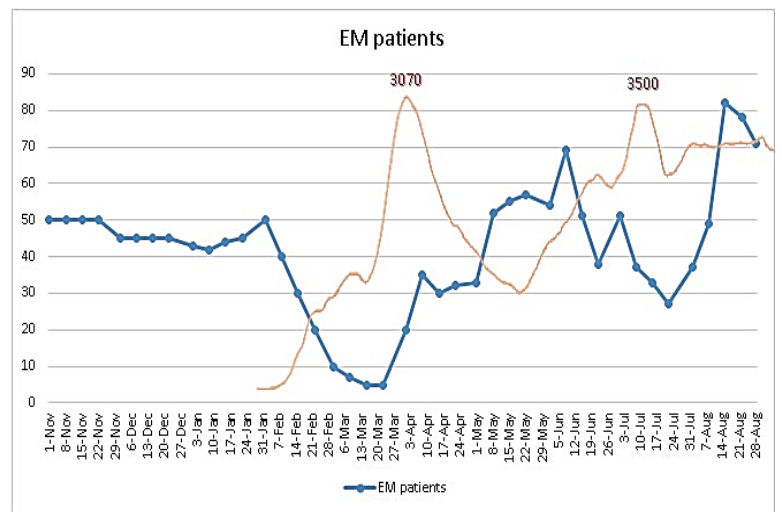

Figure 2. The mean weekly number of orthopedic patients visited in the emergency room (ER) against the chart of the daily new cases of coronavirus disease 2019 (COVID-19) in Iran

As previously discussed, our daily morning reports and educational classes were held in-person instead of virtual classes since the beginning of unlocking the department. We have a total number of 25 orthopedic residents in four levels, six of which were in level four and did not attend the hospital after March 2020. We have also three orthopedic fellowships. Before March 2020 and COVID-19 emergence, the mean daily number of residents plus fellowships and faculties attending the morning reports and educational classes were 28 and 6, respectively. During the first wave of the COVID-19 epidemic, it decreased to almost 12 and 1 , respectively, although the classes were held virtually during this time (Figure 3).

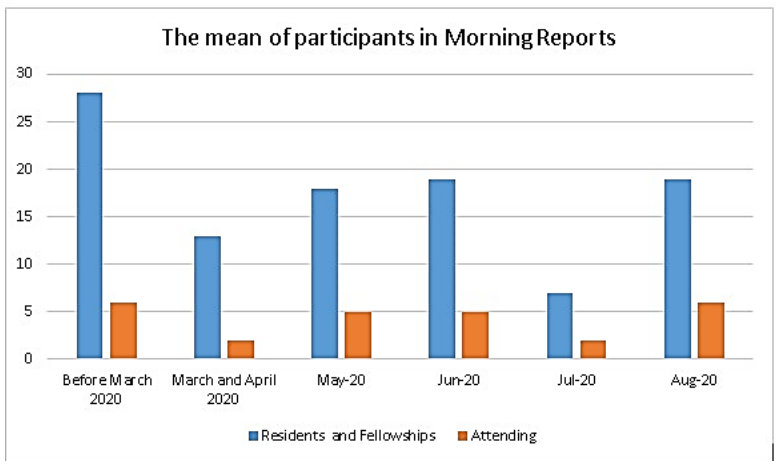

Figure 3. The mean daily number of residents, fellowships, and attendings taking part in the morning reports and educational classes

Unlocking the department, we held the classes again inperson, and the mean daily number of residents plus fellowships and faculties rose to almost 19 and 5 , respectively. However, in mid-July, most of our residents, i.e., 12 out of 19, got infected with COVID-19 and could not physically attend the classes, and as a result, the chart shows a decrease in the mean daily number of residents attending the classes to 7. Nevertheless, they could attend classes virtually. 3 out of 12 faculties were also infected and could not attend the classes in person. 


\section{COVID-19 in Residents and Staff: Our Experience}

As discussed above, in mid-July, nearly two months after the unlocking of our department, some of our residents and faculties got infected with COVID-19. We allocated a separate section to this issue because we think it might be a helpful experience for our readers, as it might happen in COVID-19 and any other similar epidemic affecting the healthcare providers in an OR. As four residents and six OR nurses got infected with COVID-19 (positive PCR test) in three consecutive days, the head of our department, after consulting with the head of infectious diseases service, in charge of the hospital COVID-19 committee, decided to screen all the residents, faculties, and OR staff for COVID-19 by PCR test. The test was done on naso- and oro-pharyngeal swabs obtained by a laboratory expert. The result was unbelievable, as 8 out of the 15 remaining residents, 3 out of 12 faculties, and 14 out of the 37 remaining OR staff, who were apparently symptomless and healthy, became positive for COVID-19. In total, 12 out of 19 residents (63.16\%), 3 out of 12 faculties (25.0\%), and 20 out of 43 OR staff (46.51\%), and in general, $50.0 \%$ of all healthcare workers were positive for COVID-19. All the PCR-positives were discharged for at least two weeks, and proper care was provided for them. It is a valuable experience as if we did not take the situation seriously and did not screen all our residents, faculties, and OR staff for COVID-19, it might infect all of them and the whole department might become locked again. However, discharging the COVID-19-positive residents, faculties, and OR staff, we kept our department working with a lower rate of operations and patients and avoided its possible re-lockdown.

\section{Discussion}

To our knowledge, there are not many studies directly discussing the unlocking of orthopedic hospital departments during the COVID-19 pandemic. In this study, our purpose was to share our experience in the unlocking process of our referral orthopedic department in a major tertiary referral hospital, which is at the same time struggling with the COVID-19 pandemic as a major referral center for COVID-19 patients. This might be a valuable experience, especially for countries with limited facilities and equipment, due to either exhaustion following the pandemic or the available local policies and circumstances.

We developed a set of local guidelines prior to unlocking our department by a team of orthopedist, anesthesiologist, and infectious disease specialist faculties of our hospital. One of the most comprehensive studies which have tried to provide guidelines regarding the resumption of elective orthopedic surgeries during the COVID-19 pandemic is the study of Parvizi et al. reporting the recommendations of the International Consensus Group (ICM) (18). This guideline emphasizes the need for screening the elective orthopedic patients for COVID-19 by means of thorough history taking and physical examination, and PCR testing whenever possible (18).

We also had the same screening guideline although we had no facility to test the patients by COVID-19 PCR routinely. We performed PCR only in those with a positive history or physical exam or when a suspicious epidemic trend of COVID-19 infection happened in our residents and OR staff. Many other of our guidelines are in complete or relative consistency with the guidelines developed by ICM, including the guidelines regarding the surgery, standard PPEs, cleaning the rooms and instruments, and postoperative care of the patients in the recovery room (18).

We reported the trend of our elective and trauma operations and ER visits from before the pandemic through the first and second wave by now. We found no similar study to compare in the literature. However, our study's disadvantage was that we could not report the number of COVID-19 infections in the follow-up of our patients due to the sudden emergence of the epidemic and the lack of an efficient follow-up registry system. We reported the number of residents and staff infected with COVID-19. In a study by Dabas et al. regarding the lockdown and unlocking of a non-COVID-19 trauma center in Delhi, India, the overall rate of healthcare workers infected with COVID-19 was $16.67 \%$, most of which was during the first week of the lockdown period. The infection rate among doctors, nurses, and other workers was $9.5 \%, 10.0 \%$, and $29.0 \%$ in their study (19). However, in our study, the overall rate was $50.0 \%$, with $63.16 \%$ of residents, $25.00 \%$ of orthopedic faculties, and $46.51 \%$ of OR staff getting infected with COVID-19. Moreover, almost all the infections happened two months after the unlocking had begun.

\section{Conclusion}

We think the unlocking of an orthopedic department in a tertiary referral hospital during the COVID-19 pandemic is a necessity, as both trauma and elective orthopedic patients need appropriate care. As mentioned above, postponing the semi-urgent and chronic elective surgeries would be troublesome for the patients, and if COVID-19 is not to be cleared soon off the community, the orthopedic field should find a way to live through it and not to be ceased, just like other medico-surgical fields. However, in advance, there is a dire need for a set of guidelines and protocols for patient care in the ward, ER, $\mathrm{OR}$, and clinic during the pandemic. The orthopedic departments can develop guidelines based on their own local policies and available facilities or modify the internationally available guidelines to their own circumstances. Moreover, we should always be cautious regarding our healthcare providers' health state during a pandemic and check them regularly for infection. With the least signs of COVID-19 infection, we should screen all the co-working healthcare workers, including the doctors, nurses, and other staff for COVID-19, in order to diagnose the silent carriers and isolate them. We should keep in mind that it can prevent the re-lockdown of our department during the unlocking period.

\section{Conflict of Interest}

The authors declare no conflict of interest in this study.

\section{Acknowledgments}

None.

\section{References}

1. Li Q, Guan X, Wu P, Wang X, Zhou L, Tong Y, et al. Early transmission dynamics in Wuhan, China, of novel coronavirus-infected pneumonia. $N$ Engl $J$ Med. 2020;382(13):1199-207.doi: 10.1056/NEJMoa2001316. [PubMed: 31995857]. [PubMed Central: PMC7121484].

2. Zhu N, Zhang D, Wang W, Li X, Yang B, Song J, et al. A novel coronavirus from patients with pneumonia in China, 2019. $N$ EngI J Med. 2020;382(8):727-33.doi: 10.1056/NEJMoa2001017. [PubMed: 31978945]. [PubMed Central: PMC7092803].

3. Ashford RU, Nichols JS, Mangwani J. Annotation:The COVID-19 
pandemic and clinical orthopaedic and trauma surgery. J Clin Orthop Trauma. 2020;11(3):504-5. doi: 10.1016/j.jcot.2020.04.002. [PubMed:32292257]. [PubMed Central: PMC7132434].

4. Sohrabi C, Alsafi Z, O'Neill N, Khan M, Kerwan A, Al-Jabir A, et al. World Health Organization declares global emergency: A review of the 2019 novel coronavirus (COVID-19). Int J Surg. 2020;76:71-6. doi:10.1016/j.ijsu.2020.02.034

5. Ajuied A, Wong F, Smith C, Norris M, Earnshaw P, Back D, et al. Anterior cruciate ligament injury and radiologic progression of knee osteoarthritis: A systematic review and meta-analysis. Am J Sports Med. 2014;42(9):2242-52. doi: 10.1177/0363546513508376. [PubMed:24214929].

6. Tuite AR, Bogoch II, Sherbo R, Watts A, Fisman D, Khan K. Estimation of coronavirus disease 2019 (COVID-19) burden and potential for international dissemination of infection from Iran. Ann Intern Med. 2020;172(10):699-701. doi: 10.7326/M200696. [PubMed:32176272]. [PubMed Central: PMC7081176].

7. Abdelnasser MK, Morsy M, Osman AE, AbdelKawi AF, Ibrahim MF, Eisa A, et al. COVID-19. An update for orthopedic surgeons. SICOT J. 2020;6:24. doi: 10.1051/sicotj/2020022. [PubMed: 32609085]. [PubMed Central: PMC7328530].

8. Askari A, Arasteh P, Jabalameli M, Bagherifard A, Razi M. COVID-19 and Orthopaedic Surgery: Experiences from Iran. $J$ Bone Joint Surg Am. 2020;102(13):1126-8. doi: 10.2106/JBJS.20.00631. [PubMed:32618911].

9. Sadighi M, Mortazavi SMJ, Ebrahimpour A, Manafi-Rasi A, Ebrahimzadeh MH, Jafari KM, et al. Fracture surgery in known COVID-19 infected patients: What are the challenges? Arch Bone It Surg. 2020;8(3):378-82. doi: 10.22038/abjs.2020.47899.2372. [PubMed: 32766396]. [PubMed Central: PMC7358239].

10. Abolghasemian $M$, Ebrahimzadeh $M H$, Enayatollahi $M$, Honarmand K, Kachooei AR, Mehdipoor S, et al. Iranian Orthopedic Association (IOA) Response Guidance to COVID-19 Pandemic April 2020. Arch Bone Jt Surg. 2020;8(Suppl 1):209-17. doi: 10.22038/ABJS.2020.47678.2370. [PubMed: 32733977]. [PubMed Central: PMC7296590].

11. Kalantar S, Farhoud A, Mortazavi J. Lockdown of an orthopedic department during COVID-19 epidemics, our experience in a general hospital. Arch Bone Jt Surg. 2020;8(Suppl1):235-41. doi: 10.22038/abjs.2020.47834.2362. [PubMed: 32607394]. [PubMed
Central: PMC7296600].

12. Khak M, Shakiba S, Rabie H, Naseramini R, Nabian MH. Descriptive epidemiology of traumatic injuries during the first lockdown period of COVID-19 crisis in Iran: A multicenter study. Asian J Sports Med. 2020;11(2):e103842. doi: 10.5812/asjsm.103842.

13. Anoushiravani AA, Barnes CL, Bosco JA $3^{\text {rd }}$, Bozic KJ, Huddleston JI, Kang JD, et al. Reemergence of multispecialty inpatient elective orthopaedic surgery during the COVID-19 Pandemic: Guidelines for a New Normal. I Bone Joint Surg Am. 2020;102(14):e79. doi: 10.2106/JBJS.20.00829. [PubMed: 32675667].

14. Graichen H. Reopening phase in Covid19 pandemic and it's changes for Orthopaedic Surgeons G-A German perspective. Orthop. 2020;20:A1-A3. doi:10.1016/j.jor.2020.08.004.

15. de Caro F., Hirschmann TM, Verdonk P. Returning to orthopaedic business as usual after COVID-19: strategies and options. Knee Surg Sports Traumatol Arthrosc. 2020;28(6):1699-704. doi: 10.1007/s00167-020-06031-3. [PubMed: 32342140]. [PubMed Central: PMC7185264]

16. Faridi S, Niazi S, Sadeghi K, Naddafi K, Yavarian J, Shamsipour $\mathrm{M}$, et al. A field indoor air measurement of SARS-CoV-2 in the patient rooms of the largest hospital in Iran. Sci Total Environ. 2020;725:138401. doi: 10.1016/j.scitotenv.2020.138401. [PubMed: 32283308]. [PubMed Central: PMC7194859].

17. World Health Organization. Cleaning and disinfection of environmental surfaces in the context of COVID-19 [Online]. [cited 2020]; Available from: URL: https://www.who.int/publications/i/item/cleaning-anddisinfection-of-environmental-surfaces-inthe-context-of-covid-19

18. Parvizi J, Gehrke T, Krueger CA, Chisari E, Citak M, Van Onsem $S$, et al. Resuming Elective Orthopaedic Surgery During the COVID-19 Pandemic: Guidelines Developed by the International Consensus Group (ICM). I Bone Joint Surg Am. 2020;102(14):1205-12. doi: 10.2106/JBJS.20.00844. [PubMed: 32675662]. [PubMed Central: PMC7431146].

19. Dabas V, Bhatia N, Goel A, Yadav V, Bajaj V, Kumar V. Management of orthopaedic accidental emergencies amidst COVID-19 Pandemic: Our experience in preparing to live with Corona. Indian J Orthop. 2020;1-6. doi: 10.1007/s43465-02000252-x. [PubMed: 32934420]. [PubMed Central: PMC7483059] 